\title{
INTERNATO EXTRACURRICULAR EM OBSTETRÍCIA NA MATERNIDADE DO HOSPITAL ESCOLA UFPEL
}

\section{EXTRACURRICULAR INTERNSHIP IN OBSTETRICS IN THE MATERNITY OF UPFEL'S TEACHING HOSPITAL}

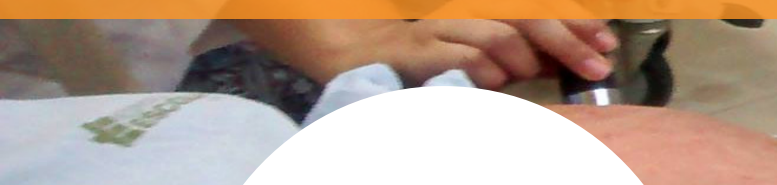

Camila Hartmann Blank ${ }^{1}$

Celene Maria Longo da Silva ${ }^{2}$

Fernanda Coutinho Kubaski ${ }^{3}$

Luísa Barin Menezes ${ }^{4}$

Renata Vernetti Giusti ${ }^{5}$

RESUMO

O projeto de ensino "Internato Extracurricular em Obstetrícia na Maternidade do Hospital Escola UFPEL" foi desenvolvido com o objetivo de inserir os estudantes já no início de sua graduação em um ambiente hospitalar. O projeto extracurricular ocorre no Hospital Escola da UFPel, e os estudantes vivenciam as rotinas práticas de uma maternidade com portas abertas 24horas, elucidando e ilustrando os conteúdos teóricos apresentados nas disciplinas curriculares do curso de medicina da UFPEL. Assim, o aluno aprende a prática da Ginecologia e Obstetrícia de maneira segura e produtiva junto aos professores e aos profissionais em formação da área.

Palavras chave: Internato extracurricular. Obstetrícia. Projeto de ensino.

\section{ABSTRACT}

The project "Extracurricular Internship in Obstetrics at the Maternity of the Hospital Escola UFPEL" was developed with the purpose of inserting the students in the beginning of their undergraduate course in a hospital environment. The extracurricular project takes place at UFPEL's Teaching Hospital, and students experience the practical routines of a maternity hospi-

\footnotetext{
${ }^{1}$ Acadêmica do Curso de Medicina da Universidade Federal de Pelotas. E-mail: blankcamila@yahoo.com.br

${ }^{2}$ Doutora em Epidemiologia pela Universidade Federal de Pelotas. Email: celene.longo@gmail.com

${ }^{3}$ Acadêmica do Curso de Medicina da Universidade Federal de Pelotas. Email: nandakubaski229@ hotmail.com
} 
tal with open doors 24 hours a day, elucidating and illustrating the theoretical contents presented curricular subjects of the medical course of UFPEL. Thus, the student learns the practice of Gynecology and Obstetrics in a safe and productive way along with the professors and professionals in training in the area.

Keywords: Extracurricular internship. Obstetrics. Project.

\section{INTRODUÇÃO}

A formação acadêmica na graduação em medicina no Brasil consiste em período de dois anos de atividades práticas orientadas chamadas de internato curricular. Os estudantes passam por todas as especialidades básicas, além de atividades de urgência e emergência. Durante o curso de medicina também são ofertadas atividades optativas, mediante processo seletivo, e são denominadas de internato extracurricular, não obrigatório, no qual o aluno pode escolher qual a área médica e em qual momento da graduação deseja realizar (CHAVES; GROSSEMAN, 2007).

O Internato é um período no qual o estudante de medicina recebe treinamento prático, sob supervisão docente, bem como entra em contato com o cotidiano de sua futura profissão, avaliando a inserção prática do aprendizado teórico recebido ao longo do curso (CHAVES; GROSSEMAN, 2007; SANTOS, 2008). Além disso, o internato é importante, pois possibilita a vivência da relação médico-paciente específica daquele local de trabalho e serve para aquisição de novas habilidades práticas, seja pelos exemplos observados ou necessidade de exercer a prática em si (GROSSEMAN; STOLL, 2008).

Salienta-se ainda que a escolha da área de especialização possa ser influenciada pelas experiências vividas durante o internato. Nesse momento, há a oportunidade de o estudante avaliar aspectos da especialidade na qual está atuando, como o manejo e a satisfação do atendimento para com os usuários do serviço assim como o estilo de vida assumido pelos profissionais da área, o que ajuda o aluno a decidir se possui ou não compatibilidade com determinada especialidade (WANDERLEY; SOBRAL, 2010).

Uma das possíveis áreas do conhecimento médico para se realizar um internato é a obstetrícia. A obstetrícia, em conjunto com a ginecologia, é uma especialidade médica que contribui para a formação de um médico generalista, que tem como uma das atribuições "promover a saúde

\footnotetext{
${ }^{4}$ Acadêmica do Curso de Medicina da Universidade Federal de Pelotas. Email: barin.luisa@gmail.com

${ }^{5}$ Acadêmica do Curso de Medicina da Universidade Federal de Pelotas.

Email: renatavernettigiusti@ hotmail.com
} 
e compreender os processos fisiológicos", bem como "realizar procedimentos clínicos e cirúrgicos indispensáveis para o atendimento inicial das urgências e emergências em todas as fases do ciclo biológico", ou seja, deve possuir conhecimento teórico e habilidades práticas para atender e promover ações de saúde em todas os ciclos da vida das mulheres, de acordo com as Diretrizes Curriculares Nacionais do Curso de Graduação de Medicina (BRASIL, 2015; BRASIL, 2017).

O Internato Extracurricular em Obstetrícia na Maternidade do Hospital Escola da UFPEL tem por meta oferecer essa vivência prática e auxiliar na aquisição de habilidades básicas na atenção integral a saúde das mulheres.

\section{METODOLOGIA}

O internato foi idealizado por um grupo de cinco acadêmicas de medicina e coordenado por uma professora de ginecologia e obstetrícia. As atividades práticas são oferecidas na modalidade de plantões de $12 \mathrm{~h}$, noturnos de segunda a sexta-feira e diurnos ou noturnos nos sábados e domingos. São oferecidas 18 vagas a estudantes matriculados a partir do $4^{\circ}$ semestre do curso de medicina da Universidade Federal de Pelotas, UFPel. Nesses plantões os alunos vivenciam a rotina prática em ambiente de atendimento hospitalar de urgências obstétricas, enfermaria de obstetrícia de risco habitual e alto risco, enfermaria de puerpério e alojamento conjunto, sala de parto e centro cirúrgico. Os internos são acompanhados por uma equipe obstétrica, formada por plantonista especialista em Obstetrícia, médico residente e alunos do internato curricular. $\mathrm{O}$ internato extracurricular foi idealizado com o objetivo geral de ensinar a rotina de atendimento em maternidade, inserindo alunos dos semestres iniciais no atendimento obstétrico do Hospital Escola da UFPEL. Os objetivos específicos são colocar em prática o conhecimento do transcorrer fisiológico e patológico durante a gestação, parto e puerpério; identificar sinais de trabalho de parto prematuro e a termo; identificar as principais patologias da gestação que necessitam de atendimento hospitalar; acompanhar o trabalho de parto, parto e puerpério das gestantes atendidas no serviço; identificar as complicações puerperais que levam a atendimento hospitalar, clínico ou cirúrgico; integrar os alunos da graduação com a equipe de plantão obstétrico.

O objetivo desse relato é descrever as experiências dos acadêmicos do curso de medicina no Hospital Escola da UFPel no Projeto de Ensino "Internato Extracurricular em Obstetrícia na Maternidade do Hospital Escola - UFPel". Os acadêmicos passam a integrar o internato extracurricular após terem sido selecionados por meio de uma prova escrita, onde aqueles que obtiveram as melhores notas foram incluídos na lista de in- 
ternos e passam a constar em uma escala de plantão obstétrico. Também são incluídos no projeto de ensino no sistema de registro acadêmico da UFPEL. Alguns alunos pelos mais diversos motivos desistem antes de completar os seis meses previstos, com isso os suplentes serão chamados para preencher as vagas remanescentes.

Nos primeiros plantões, os novos integrantes recebem tutoria dos internos mais antigos, dos médicos residentes e do preceptor do plantão sobre as normas de funcionamento do serviço e de todas as rotinas de atendimento na maternidade. Inicialmente as atividades são de observação e pequenas inserções na atenção, após alguns plantões recebem incumbências mais elaboradas, sempre com a supervisão dos seus colegas de plantão. Em todos os plantões há a presença de um médico residente em obstetrícia e ginecologia e de acadêmicos de medicina em internato curricular.

Os cerificados de participação serão elaborados perante a comprovação da frequência aos plantões, por isso as presenças dos acadêmicos nos plantões são registradas em um caderno específico para essa finalidade. A partir do momento em que cada um que tem seu nome na escala, o mesmo responde pela tarefa daquele dia e horário. Eventuais trocas de plantão deverão ser registradas no site do internato e visualizado pela pessoa responsável pela liberação da escala, caso ocorra alguma falta devido a qualquer mal entendimento nas trocas a responsabilidade será de quem estava originalmente escalado e, não havendo justificativa documentada e relevante, o interno será excluído do grupo.

Além do plantão obstétrico, os alunos participantes do internato também podem participar de atividades teóricas complementares de outro projeto de ensino, a Liga Acadêmica de Ginecologia e Obstetrícia da UFPEL, que realiza encontros quinzenais para seminários, discussão de artigos ou aulas com professores convidados. Ambos os projetos de ensino têm autorização para funcionar no Hospital Escola da UFPEL quanto no Colegiado do Curso de Medicina.

As imagens aqui apresentadas foram liberadas para publicação tanto por parte dos alunos envolvidos quanto pelas gestantes atendidas, mediante consentimento informado.

\section{RESULTADOS E DISCUSSÃO}

A partir da participação no Internato Extracurricular de Obstetrícia na Maternidade do Hospital Escola UFPEL, os alunos são estimulados a desenvolver capacidade de diálogo sobre atenção às gestantes, parturientes e puérperas, participando da realidade de um hospital de pequeno a médio porte, ligado a uma instituição federal de ensino (Figura 1). Diante disso, há um maior conhecimento acerca da especialidade de ginecologia 
e obstetrícia, da relação médico-paciente em nível ético e moral e da realidade de um hospital público, o que ajudará na formação de um médico mais consciente e humano (GROSSEMAN; STOLL, 2008).

Fachada do Hospital Escola UFPel.

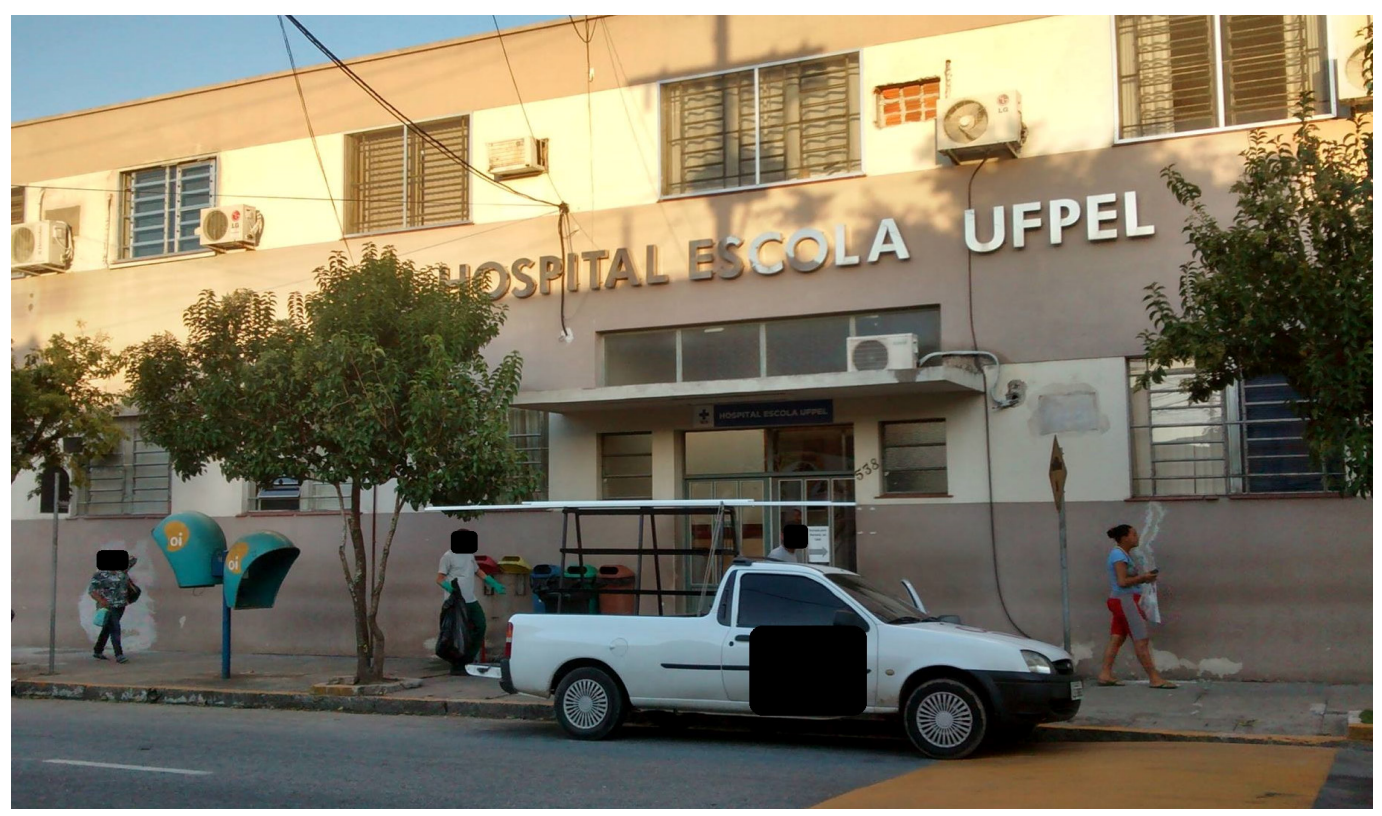

Fonte: Fotografia de Renata Vernetti Giusti, 2016.

As habilidades e competências dos alunos são consolidadas por meio da realização da anamnese e do exame físico das gestantes que procuram atendimento na Maternidade do Hospital Escola UFPEL, exemplificado nas Figuras 2, 3 e 4, gerando um aprofundamento nos conhecimentos de fisiologia e patologias específicas da gestação, que serão essenciais à prática médica e contribuirão para a formação de melhores profissionais (BRASIL, 2015; BRASIL, 2017). Ainda há a melhora na qualidade e na agilidade do atendimento prestado na maternidade, já que a inserção de alunos em semestres mais iniciais promovem constantes questionamentos sobre as práticas rotineiras. Isso poderá também identificar necessidade de atualizações e de treinamento nas habilidades de ensino em diferentes níveis de conhecimento sobre a prática da obstetrícia, seja tanto por parte dos médicos residentes quanto dos preceptores (BRASIL, 2015; BRASIL, 2017). 


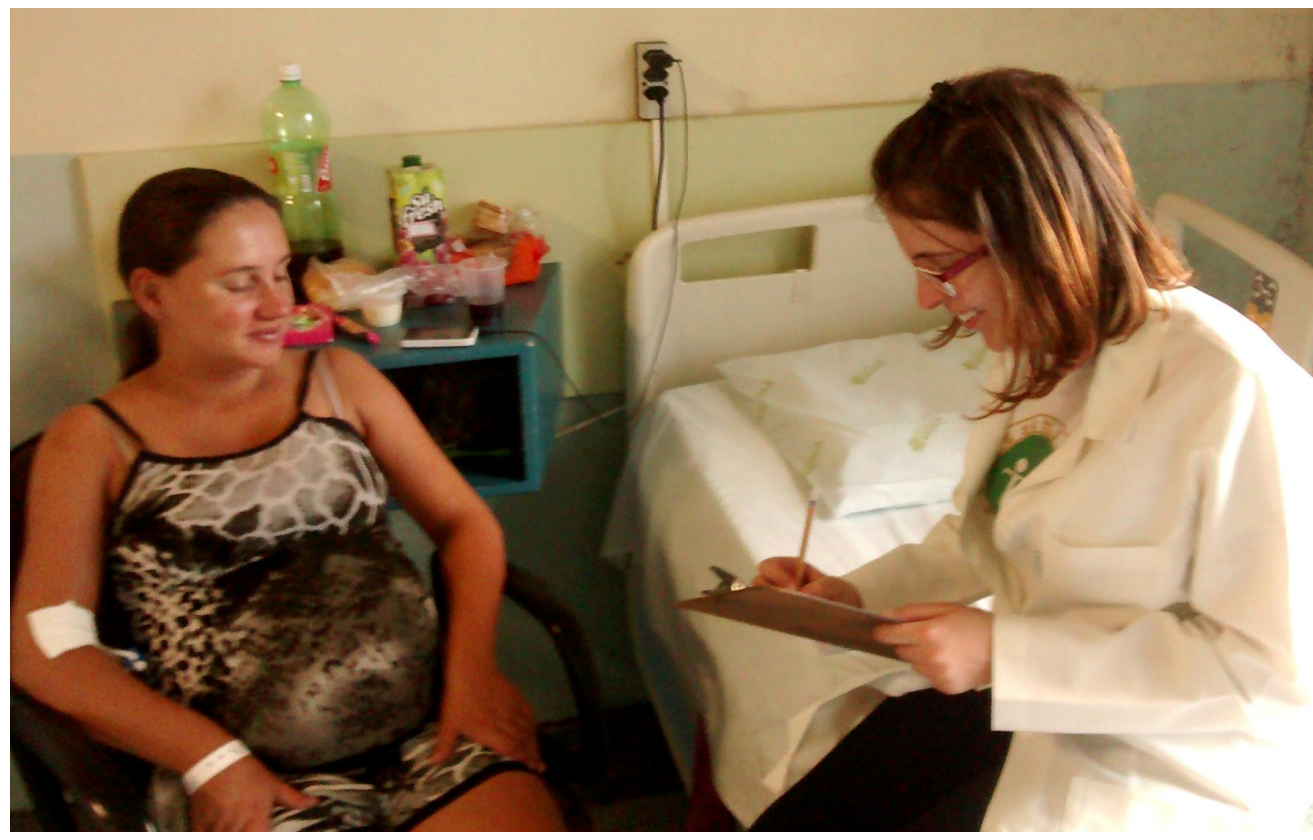

Fonte: Fotografia de Renata Vernetti Giusti, 2016.

*Permissão para publicação da imagem arquivada com a autora.

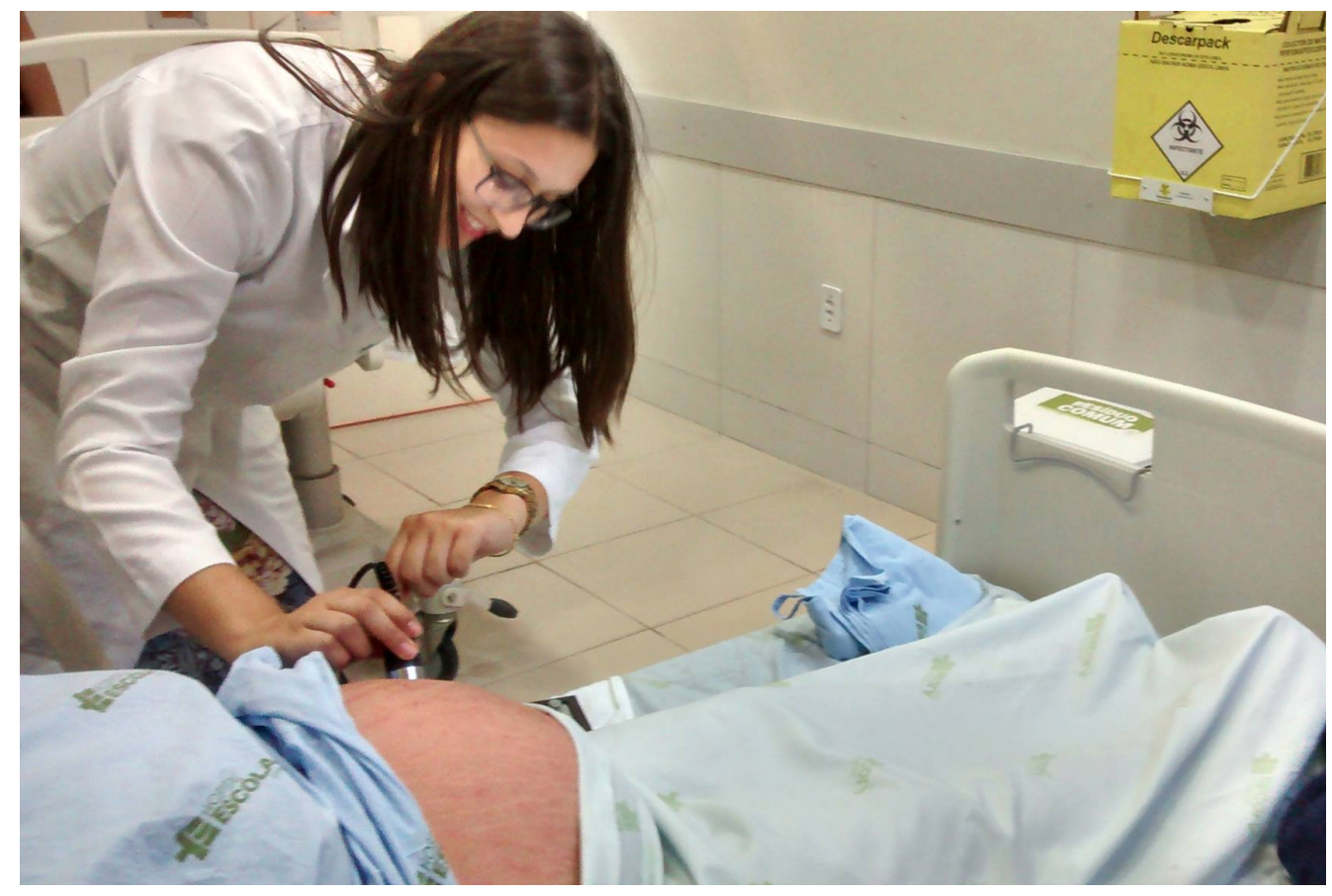

Fonte: Fotografia de Renata Vernetti Giusti, 2016.
Figura 2

Fotografia de uma Interna realizando anamnese com uma paciente.

Figura 3

Interna realizando a aferição dos batimentos cardíacos fetais em paciente. 


\section{8 - BLANK, H. C. et al.}

Figura 4

Fotografia de interna realizando exame de bem-estar fetal.

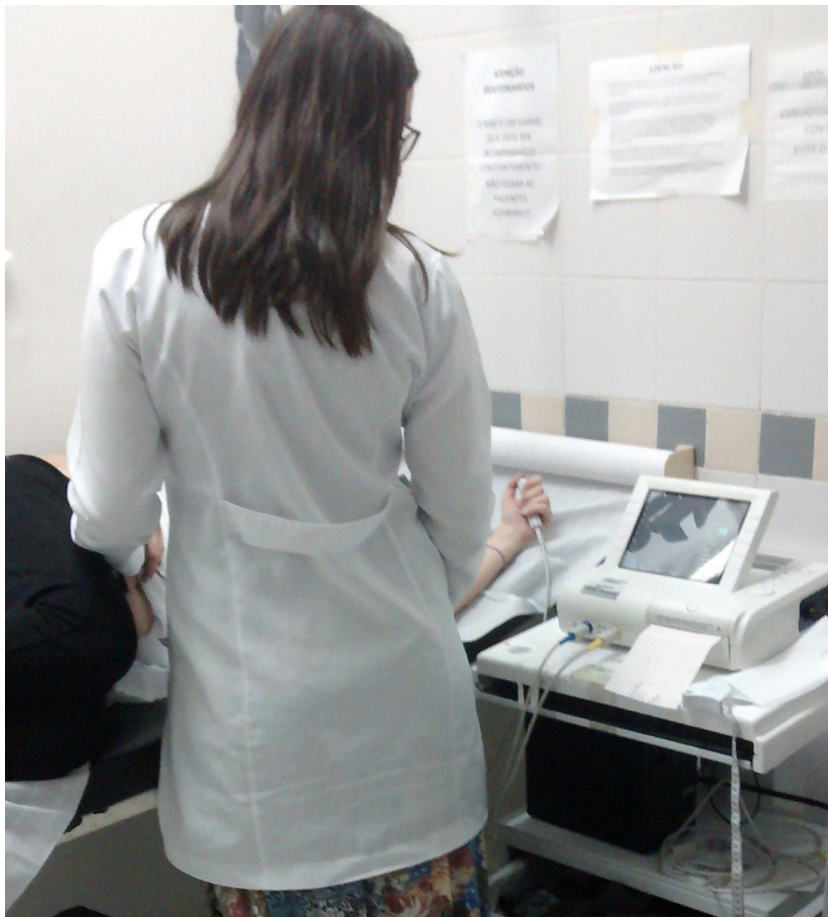

Fonte: Fotografia de Renata Vernetti Giusti, 2016.

Tal aprimoramento dos conhecimentos do aluno só é possível graças à eficiente equipe de trabalho da Maternidade do Hospital Escola UFPel. Na Figura 5 estão representadas duas internas extracurriculares, a preceptora do plantão, médicas residentes do terceiro e primeiro ano e dois internos regulares. Os preceptores e residentes consideram importante transmitir ensinamentos teóricos e práticos aos alunos, possibilitando uma aprendizagem supervisionada ao mesmo tempo em que estimulam a valorização da presença dos alunos que optaram por estagiar naquele ambiente de atividades práticas. $O$ hospital disponibiliza a estrutura necessária para comportar tal número de alunos nos plantões, com ambiente espaçoso e limpo, além de acesso à cantina para alimentação. 


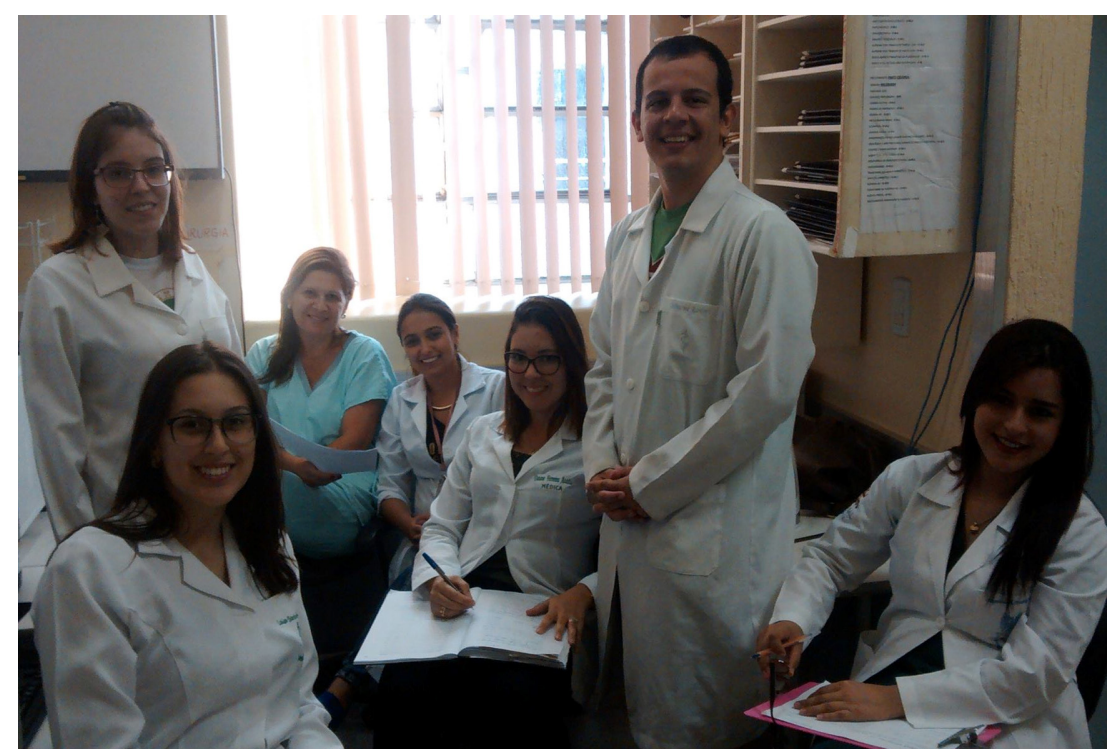

\section{Figura 5}

Fotografia da equipe com os internos.

Fonte: Fotografia de Renata Vernetti Giusti, 2016.

Outro benefício da participação do Internato é a ajuda na escolha de uma especialidade médica através da vivência de um ambiente de ensino e aprendizagem simultâneo ao serviço de assistência na área de ginecologia e obstetrícia. Participando do Internato, com experiências positivas (Figura 6), situações angustiantes e até mesmo alguns momentos dramáticos com resultados negativos, os alunos são levados a questionar constantemente sobre as escolhas para o seu futuro, bem como sobre as melhores evidências científicas para as práticas diárias. Outra possibilidade de realização no ambiente da maternidade poderá ser o desenvolvimento de pesquisas em ambiente hospitalar, que podem ampliar o conhecimento dos estudantes envolvidos e contribuir com o meio científico e com a comunidade, uma vez que estratégias de atendimento e de prevenção de doenças e de desfechos negativos na gestação podem ser elaboradas através desses estudos. 
Figura 6

Fotografia de interna com recém-nascido na Maternidade.

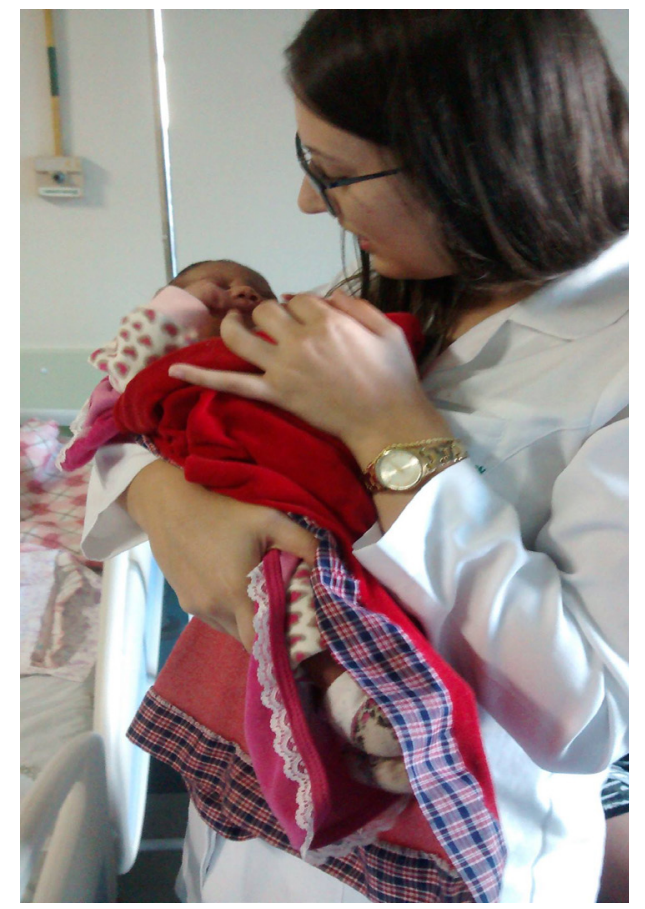

Fonte: Fotografia de Renata Vernetti Giusti, 2016.

Como desafio para melhoria no internato, fica a sugestão de que antes do início do de nova turma haja uma reunião com os futuros internos, com o intuito de deixar claros todos os objetivos do internato e os deveres do interno, além de apresentar os mecanismos de funcionamento da Maternidade do Hospital Escola UFPEL. Logo, o interno, ao entender o seu papel, terá a possibilidade de realizar um trabalho mais eficiente e o organizado, obtendo maior conheço acerca das rotinas em ginecologia e obstetrícia.

Além disso, recomenda-se uma reunião de retorno individual, na qual o preceptor possa avaliar o desempenho do aluno e que este possa dissertar sobre suas impressões acerca do internato. Assim, seria possível proporcionar maior crescimento aos estudantes que não estejam enquadrados à proposta do projeto, corrigindo os erros e reforçando os acertos, bem como desenvolver mudanças que visem a melhorias no sistema de aprendizagem e no atendimento às mulheres.

\section{CONCLUSÃO}

Com a inserção do estudante na maternidade do Hospital Escola da UFPel houve a identificação de diversas contribuições positivas desse momento para a educação médica, como, por exemplo, a aplicação prática dos conhecimentos teóricos de ginecologia e obstetrícia. Além disso, a demanda dos atendimentos realizados pelos acadêmicos de medicina 
mostra a contribuição desses ao Sistema Único de Saúde, assim como ao Programa de Residência Médica em Obstetrícia e Ginecologia do HE UFPEL.

Assim, pode-se concluir que a inserção dos acadêmicos de semestres mais iniciais em um estágio extracurricular pode ampliar em grande parte o seu conhecimento tanto na construção mais sólida da sua educação médica, quanto para descobrir na prática os aspectos positivos e negativos no âmbito da assistência em saúde pública à população.

\section{REFERÊNCIAS}

BRASIL. Ministério da Educação. Diretrizes Curriculares Nacionais do Curso de Graduação em Medicina, 2001. Resolução CNE/CES № 4, de 7 de novembro de 2001. Disponível em: <http://portal.mec.gov.br/cne/arquivos/pdf/CES04.pdf>. Acesso em: 14 mar. 2015.

BRASIL. Ministério da Educação. Diretrizes Curriculares Nacionais do Curso de Graduação em Medicina. Resolução № 3, de 20 de junho de 2014. Disponível em: <http://portal.mec.gov.br/index.php?option=com_ docman\&view=download\&alias $=15874$ rces0034\&category_slug=junho2014pdf\&ltemid=30192> . Acesso em: 15 maio 2017.

CHAVES, I. T. S.; GROSSEMAN, S. O internato médico e suas perspectivas: estudo de caso com educadores e educandos. Rev. Bras. de Educ. Med., v. 31, n. 3, p. 212-222, 2007.

GROSSEMAN, S.; STOLL, C. O Ensino-aprendizagem da relação médico-paciente: estudo de caso com estudantes do último semestre do curso de Medicina. Rev. Bras. de Educ. Med., v. 32, n. 3, p. 301-308, 2008.

SANTOS, S. S. et al. Avaliação da progressão cognitiva no internato de clínica médica. Rev. Bras. de Educ. Med., v. 32, n. 3, p. 390-395, 2008.

WANDERLEY, M. S.; SOBRAL, D. T. Escolha da especialização em ginecologia e obstetrícia. Rev. Bras. Ginecol. Obstet., v. 32, n. 1, p. 55-56, 2010.

Data de recebimento: 16 de março de 2016.

Data de aceite para publicação: 17 de maio de 2017. 